\title{
BMJ Open Effectiveness of complex multiple-risk lifestyle interventions in reducing symptoms of depression: a study protocol for a systematic review and meta-analysis of randomised controlled trials
}

\author{
Irene Gómez-Gómez, ${ }^{\circledR}$ Emma Motrico, ${ }^{1,2}$ Patricia Moreno-Peral, 2,3,4 \\ Alina Rigabert, ${ }^{1}$ Sonia Conejo-Cerón, ${ }^{2,3,4}$ Manuel Ortega-Calvo, ${ }^{5,6}$ \\ José-Ignacio Recio, ${ }^{2,7}$ Juan A Bellón ${ }^{2,3,4,8,9}$
}

To cite: Gómez-Gómez I, Motrico E, Moreno-Peral P, et al. Effectiveness of complex multiple-risk lifestyle interventions in reducing symptoms of depression: a study protocol for a systematic review and meta-analysis of randomised controlled trials. BMJ Open 2019;9:e026842. doi:10.1136/ bmjopen-2018-026842

- Prepublication history and additional material for this paper are available online. To view these files, please visit the journal online (http://dx.doi. org/10.1136/bmjopen-2018026842).

Received 23 September 2018 Revised 11 January 2019 Accepted 25 January 2019

Check for updates

(C) Author(s) (or their employer(s)) 2019. Re-use permitted under CC BY-NC. No commercial re-use. See rights and permissions. Published by BMJ.

For numbered affiliations see end of article.

Correspondence to

Dr Emma Motrico;

emotrico@uloyola.es

\section{ABSTRACT}

Introduction Many studies have explored the impact of lifestyle interventions on depression. However, little is known about the effectiveness of complex multiple-risk lifestyle interventions in reducing symptoms of depression. Our objective is to assess the effectiveness of complex multiple-risk lifestyle interventions in reducing depressive symptoms in the adult population by the acquisition of at least two healthy habits-healthy diet, physical activity and/or smoking cessation. For such purpose, a systematic review and meta-analysis of randomised controlled trials will be conducted.

Method and analysis MEDLINE (through Ovid and PubMed), Scopus, Cochrane Central Register of Controlled Trials, Web of Science, PsycINF0, OpenGrey Register (System for Information on Grey Literature in Europe) and the International Clinical Trials Registry Platform will be searched for relevant articles. Additionally, a supplementary manual search will be performed using lists of references, references to expert authors and other systematic reviews and/or meta-analyses. Study selection, data extraction (target habits, country, target populations, conditions and statistical data to name a few) and assessment of the risk of bias will be performed separately by two independent researchers. The primary outcome measure will be the reduction of depression symptoms, as measured by validated instruments. We will calculate pooled standardised mean differences and $95 \%$ Cls using random-effect models. Heterogeneity, sensitivity and publication bias will be assessed, and sub-group analysis will be performed. Heterogeneity will be explored by random-effects meta-regression analysis.

Ethics and dissemination Ethical approval is not required for this study. The results of this systematic review and meta-analysis will be presented in relevant conferences and published in a peer-review journal. The findings of this study could have important clinical and scientific implications for the improvement of symptoms of depression.

\section{Strengths and limitations of this study}

- This is the first systematic review and meta-analysis to assess the effectiveness of complex multiple-risk lifestyle interventions in reducing depressive symptoms by the promotion of healthy habits in the adult population.

- This study will be conducted in accordance with Preferred Reporting Items for Systematic Reviews and Meta-Analyses statement for reporting systematic reviews and meta-analyses.

- The effect size, robustness and quality of evidence of the studies included in this meta-analysis will help to determine whether symptoms of depression can be improved by complex multiple-risk lifestyle interventions based on the acquisition of healthy habits.

- This study will have the limitations inherent to all systematic reviews and meta-analyses (ie, missing outcome data, methodological flaws and risk of bias of the studies included).

- Bias, heterogeneity and the small sample of studies could limit the interpretation of results.

PROSPER0 registration number CRD42018100253; Pre-results.

\section{INTRODUCTION}

Depression affects more than 300 million people worldwide and is the first global cause of disability. ${ }^{1}$ Some studies have shown that pharmacological and psychological treatments reduce disease burden by less than $30 \% .^{2}$ Many randomised controlled trials (RCTs) and systematic reviews have been conducted to assess the efficacy of different interventions in the prevention of depression, 
the reduction of new episodes ${ }^{3-5}$ or the improvement of depressive symptoms. ${ }^{67}$

Recent compelling evidence suggests that depression has a significant lifestyle-driven component. ${ }^{8}$ It is increasingly accepted that poor sleep, dietary habits, smoking and sedentary habits are important modifiable risk factors for depressive disorders. ${ }^{9-12}$ Nowadays, the most prevalent lifestyle risk factors in many countries include tobacco use, physical inactivity and unhealthy diet. ${ }^{13-16}$

In most studies, lifestyle interventions for the prevention of depression are only focused on a specific healthy habit. ${ }^{17-26}$ Nevertheless, unhealthy lifestyle factors tend to co-occur; it has been reported that $31 \%-40 \%$ of the population has at least two risk factors. ${ }^{14}{ }^{27}$ Evidence suggests that complex multiple-risk lifestyle interventions provide greater health benefits, reduce costs and optimise the promotion of healthy habits. ${ }^{28}$

According to our search, only three systematic reviews and meta-analyses have explored the effect of multifactorial lifestyle interventions on symptoms of depression. One of these studies ${ }^{29}$ was focused on both single and multifactorial lifestyle interventions promoting healthy diet and physical activity in high-risk or type-2 diabetes patients. The other study ${ }^{30}$ only evaluated a lifestyle/ psychologicalintervention versus a multi-component intervention based on a combination of lifestyle and psychological interventions in patients with chronic obstructive pulmonary disease. The third study ${ }^{31}$ assessed the effects of dietary interventions and complex multiple-risk lifestyle interventions. Thus, most studies were focused on dietary and physical activity interventions addressed at a specific population. Some complex interventions combined a psychological intervention with an intervention on a specific lifestyle habit. ${ }^{30}$

To the best of our knowledge, no systematic review or meta-analysis has been performed so far to explore the effectiveness of complex multiple-risk lifestyle interventions in reducing depressive symptoms in the adult population.

A systematic review and meta-analysis of RCTs will be conducted. The objective is to assess the effectiveness of complex multiple-risk lifestyle interventions in reducing depressive symptoms in the adult population by the promotion of at least two healthy habits (healthy diet, physical activity and/or smoking cessation). The research question of this study will be: Are complex multiple-risk lifestyle interventions effective in improving symptoms of depression in the adult population? This study will help to understand if symptoms of depression improve with the implementation of complex multiple-risk lifestyle interventions promoting the acquisition of healthy habits. The expected results will have multiple implications, since complex multiple-risk lifestyle interventions prevent other non-communicable diseases ${ }^{32}$ reduce medical costs $^{33}$ and improve self-efficacy in patients. ${ }^{34}$ Furthermore, complex multiple-risk lifestyle interventions respond accurately to clustering of unhealthy lifestyle factors ${ }^{14} 27$ and can easily be implemented in different settings. ${ }^{26} 323536$

\section{METHODS AND ANALYSIS}

This protocol was designed in accordance with the 2015 Preferred Reporting Items for Systematic Reviews and Meta-Analyses Protocols. ${ }^{37}$ The protocol was registered with the International Register of Systematic Reviews (http://www.crd.york.ac.uk/PROSPERO) on 18 September 2018.

\section{Eligibility criteria}

The rationale for the inclusion and exclusion criteria (using PICOS acronym ${ }^{38}$ ) was to select RCTs (S) assessing the effectiveness of complex multiple-risk lifestyle interventions (I) - as compared with usual care (ie, staying in the waiting list, no intervention or joining an attention control group $[\mathrm{C}]$ ) - in promoting healthy behaviours to reduce symptoms of depression $(\mathrm{O})$ in the adult population $(\mathrm{P})$. Table 1 presents the inclusion and exclusion criteria.

\section{Study design}

Only randomised control and clinical trials will be included, since according to the 'hierarchy of evidence', these designs provide the best evidence for healthcare interventions. ${ }^{39}$ In relation to cluster randomised control/clinical trials, we will only include those with at least two clusters per intervention and per control group, with analysis of effectiveness was adjusted for the clustering effect. Observational studies, non-RCTs and before-after studies will be excluded. Concerning more than two-arm interventions, we will only include the arms that meet our inclusion criteria.

\section{Participants, setting, time frame and language}

We will consider the entire adult population, with no restrictions in terms of disease or health status (ie, healthy people and patients with chronic diseases, among others). Participants must be 18 years or older. No other sociodemographic restrictions will be imposed. All settings will be considered and no time frame or language restrictions will be applied to the literature search. In purely preventive intervention studies, baseline depression must have been discarded using a standardised diagnostic tool or a threshold in a validated scale. In relation to inclusion criteria, as it is unlikely that baseline depression was assessed in RCTs to exclude depressive patients, it will only be required that baseline symptoms of depression were assessed. Baseline depression scores will be considered to clearly distinguish between different types of prevention interventions. Studies which target population includes only patients meeting the diagnostic criteria for clinical depression will be excluded, as these are considered treatment rather than preventive interventions.

\section{Type of intervention}

We will include complex multiple-risk lifestyle interventions, defined as interventions or programmes promoting the acquisition of at least two of the following healthy habits: healthy diet, physical activity and/or smoking cessation. Quantitative and qualitative approaches will be 


\begin{tabular}{|c|c|c|}
\hline Criteria & Inclusion criteria & Exclusion criteria \\
\hline Population & Adults over 18 years of age. & $\begin{array}{l}\text { Studies which target population are patients meeting the } \\
\text { diagnostic criteria for clinical depression. }\end{array}$ \\
\hline Intervention & $\begin{array}{l}\text { Complex multiple-risk lifestyle interventions addressing } \\
\text { at least two of the following factors: healthy diet, physical } \\
\text { activity and smoking cessation*. }\end{array}$ & $\begin{array}{l}\text { Any other intervention that was not a complex multiple- } \\
\text { risk lifestyle intervention. }\end{array}$ \\
\hline Outcome & Reduction of symptoms of depression. & $\begin{array}{l}\text { Interventions which effects on depression or other } \\
\text { diseases are not reported separately (or which effective } \\
\text { measures are not provided separately). }\end{array}$ \\
\hline Study design & $\begin{array}{l}\text { Randomised controlled trials, randomised clinical trials or } \\
\text { clinical trials }\end{array}$ & $\begin{array}{l}\text { Prospective cohort, retrospective cohort, cross-sectional, } \\
\text { case-control, systematic reviews and meta-analysis, } \\
\text { protocols, clinical case, editors' letters, qualitative } \\
\text { studies and observational studies }\end{array}$ \\
\hline Setting & All settings & None \\
\hline
\end{tabular}

*Interventions or programmes addressing at least two of the following factors: healthy diet, physical activity and/or smoking cessation. Healthy diet: Mediterranean diet, Dietary Approaches to Stop Hypertension diet, Japanese diet, any diet with a goal of five pieces of fruit and vegetables per day and any diet where saturated fats account for less than $10 \%$ of the total daily energy intake. Physical activity: At least 150 minutes of moderate-intensity aerobic physical activity throughout the week, or at least 75 minutes of vigorous-intensity aerobic physical activity throughout the week, or an equivalent combination of moderate-intensity and vigorousintensity activities. Smoking cessation: Any smoking cessation intervention with a self-reported and/or biochemically validated abstinence outcome measure of continuous abstinence, and/or prolonged abstinence, and/or point prevalence; and studies where treatment effect size is reported based either on the absolute percentage difference between abstinent subjects and smokers or the magnitude of the difference between active and control conditions.

used for evaluating healthy diet interventions. Healthy diets include the Mediterranean diet, ${ }^{40}{ }^{41}$ the Dietary Approaches to Stop Hypertension diet ${ }^{42}{ }^{43}$ the Japanese diet, ${ }^{445}$ any diet with a target of five pieces of fruit and vegetables per day ${ }^{46}$ and any diet where saturated fats account for less than $10 \%$ of the total daily energy intake. ${ }^{47} 48 \mathrm{In}$ relation to physical activity, we will consider interventions that involve at least 150 or 75 minutes of moderate or vigorous aerobic exercise, respectively, per week, or an equivalent time where moderate and vigorous activities are combined. ${ }^{49}$ Smoking cessation interventions will be required to include a self-reported and/or biochemically validated outcome of continuous abstinence, and/ or prolonged abstinence, and/or point prevalence. In addition, we will include studies where treatment effect size is expressed as the absolute percentage difference between abstinence and smoking or the magnitude of the difference between active and control interventions. For continuous abstinence, any smoking between the quit day and the end of the follow-up will be considered a failure. Failure of prolonged abstinence will be defined as smoking seven consecutive days or at least once a week for two consecutive weeks after the quit day. Failure of point prevalence will be defined as any smoking 7 days prior to the follow-up. ${ }^{50}$ Studies with pharmacological therapy for smoking cessation will be excluded.

\section{Comparators}

Comparator groups will include a usual care group, a waiting list group, a non-intervention group (patients undergo the same assessments as the treatment group, but were not engaged in any intervention) and attention controls (active control or placebo).

\section{Outcome}

We will only include RCTs which primary or secondary outcome measure is variance in symptoms of depression. Outcomes must have been measured using validated rating scales for depression. If more than one scale was used to assess depression, only the results of the scale with the best psychometric properties for the setting and country will be used. If this information is not provided by the authors, a literature search will be conducted to find it out. When several scales have been used, the parameters used to select the best scale are: a higher Youden's $\mathrm{J}$ statistic (J=Sensitivity+Specificity-1), Cronbach's alpha and intraclass correlation coefficient (test-retest); and sensitivity to change over time (Yes/no/not available). Studies, where effects on depression and other mood disorders (eg, anxiety) are not provided separately, will be excluded.

Outcomes will have been addressed during the post-intervention period and the last follow-up visit of the study. When available, we will consider the average of all the follow-up visits.

\section{Information resources and search strategy}

A literature search of the following electronic databases will be performed: MEDLINE (via Ovid and PubMed), Scopus, Cochrane Central Register of Controlled Trial, 
PsycINFO, Web of Science, OpenGrey Repository (System for Information on Grey Literature in Europe) and the International Clinical Trials Registry Platform. Additionally, an online supplementary file manual search will be performed using lists of references, references to expert authors and other systematic reviews and/or meta-analyses of multiple or single lifestyle interventions focused on healthy diet, physical activity or smoking cessation. The search strategy will involve the use of a set of terms selected from the relevant literature. We will use Medical Subject Headings and text words related to healthy diet, physical activity, smoking cessation and depression. The search will be first performed in MEDLINE and will subsequently be adapted to the other relevant databases. The time frame of the search will extend until the review is completed. The online supplementary file shows Medline's search strategy using the PICOS format in PubMed. No limits will be imposed on study language or publication date.

\section{Study selection}

The study selection process will be performed in three phases: first, duplicated records will be eliminated; then a selection of titles and abstracts will be conducted; finally, only selected records will be read full-text. The whole process will be completed twice by two independent researchers. Discrepancies will be solved by consensus between the two reviewers; if the consensus is not reached, a third reviewer will make the final decision. The level of agreement between researches will be calculated using Kappa index. ${ }^{51}$

\section{Data extraction}

An evidence table will be generated to display the most relevant characteristics of each study. If several records of the same study are found, they will be computed as a single study, and data extraction will be made all at once using information from all the records. Paper screening and selection, and data extraction will be performed by two independent reviewers twice. Discrepancies will be solved by a third reviewer. In case of missing data, authors will be contacted. The following qualitative data will be collected: authors, year of publication, country, primary outcome, target population, target habits (healthy diet, physical activity and/or smoking cessation), characteristics of the complex intervention (total duration of the intervention, framework and session format), conditions, sample size (control/intervention), deliverer, outcome (measuring instrument), adherence to healthy habits at the end of the intervention and setting and duration of the follow-up.

Regarding quantitative data, we will extract means and $\mathrm{SD}$, when available, to calculate the standardised mean difference (SMD). If some RCTs report other formats, Comprehensive Meta-analysis (CMA) will be used to obtain the equivalent SMD.

\section{Risk of bias}

The internal validity of the selected studies will be assessed using the Cochrane Collaboration's tool for assessing risk of bias. ${ }^{52}$ This tool assesses six general dimensions or criteria: selection bias (random sequence generation and allocation concealment), performance bias (blinding of participants and trial personnel), detection bias (blinding of evaluators of outcome assessment), attrition bias (missing outcome data), reporting bias (selective reporting) and other biases (other sources of bias). In all items, zero points are assigned for low risk of bias, one point for unclear risk and two points for high risk. The risk of bias will be assessed twice by two independent reviewers. Discrepancies will be solved by a third reviewer. The level of agreement between researches will be calculated using the intraclass correlation coefficient. ${ }^{51}$ If additional information is required, authors will be contacted.

\section{Assessment of publication bias}

Publication bias will be assessed by a funnel plot. Duval and Tweedie's trim and fill procedure ${ }^{53}$ will be used to impute missing data for the analysis. If the number of studies is equal or greater than 10, Begg and Mazumdar rank correlation $^{54}$ and Egger's test ${ }^{55}$ will also be performed.

\section{Statistical analysis}

Two independent reviewers will insert quantitative data from each study twice into an Excel sheet. If there are missing data that hinder effect size calculation, the authors will be contacted. If no response is received, the study will be excluded. We will use CMA to convert data into the desired format. Quantitative data will be analysed using CMA V.3 and STATA V.14. Effects size will be calculated using SMD and 95\% CI. A negative SMD will be interpreted as a greater reduction of depressive symptoms in the intervention group. Cohen's interpretation will be used-that is, $0.20,0.50$ and 0.80 as small, medium and large effect size, respectively. ${ }^{56}$ We selected a random-effects model for our meta-analysis, under the assumption that the RCTs to be included in our study will have been performed in heterogeneous populations that may differ from each other.

Heterogeneity will be assessed using forest plots, $Q$ statistics, $\mathrm{p}$ values and $\mathrm{I}^{2}$ index values and their $95 \%$ CIs. $\mathrm{I}^{2}$ index values will be expressed as percentages and interpreted as follows: unimportant heterogeneity $(0 \%-40 \%)$, moderate heterogeneity $(30 \%-60 \%)$, substantial heterogeneity $(50 \%-90 \%)$ and considerable heterogeneity $(75 \%-100 \%))^{38}$

Sensitivity will be assessed during the first and last follow-up visit using fixed-effects model and Hedges' g. Some studies will be excluded from analysis (eg, studies with the highest risk of bias or causing the greatest increase in heterogeneity).

Sub-group analysis will be performed to explore heterogeneity across studies. When available, the following data will be collected: year of publication, country, primary outcome, target population, target habits (healthy diet and physical activity; healthy diet and smoking cessation; physical activity, smoking cessation and healthy diet; and physical activity and smoking cessation), severity of 
depressive symptoms at baseline, population age range, comparator (usual care, waiting list, non-intervention and attention controls), sample size, setting (ie, hospital, community hubs, school and workplace), characteristics of the complex intervention (total duration of the intervention, framework and session format), follow-up (shortterm vs long- term), deliverer, and risk of bias.

Random-effects meta-regression will be used to identify differences in effect sizes in relation to risk of bias or time. Normality of distribution will be previously assessed by the Skewness and Kurtosis normality test. ${ }^{57}$ When appropriate, quantitative variables will be transformed to improve approximation to normality. Covariables with $p$ values less than 0.15 , which were not previously removed from the model due to collinearity, will be included in meta-regression models. CIs and standard errors will be calculated by the Knapp and Hartung method. ${ }^{58}$ We will also use Higgins and Thompson permutation test to calculate $p$ values, taking multiplicity adjustment into account. ${ }^{59}$ We will use a plot of standardised shrunken residuals to test goodness of fit in meta-regression models.

\section{Quality of evidence}

Quality of evidence will be assessed using Grading of Recommendations Assessment, Development and Evaluation (GRADE) system. GRADE system helps to determine if the estimated effect size is reliable or not as defined according to four categories: high, moderate, low and very low. ${ }^{60}$

\section{Patient and public involvement}

No patients or public involved.

\section{ETHICS AND DISSEMINATION}

This systematic review and meta-analysis study will be published in a peer-reviewed journal. Results will be presented in relevant international conferences. Due to the characteristics of this study, no ethical approval is required.

\section{DISCUSSION}

To the best of our knowledge, this will be the first systematic review and meta-analysis to assess the effectiveness of complex multiple-risk lifestyle interventions (healthy diet, physical activity and/or smoking cessation) in reducing depressive symptoms in the adult population. In this study, we will summarise and evaluate the quality of the most relevant studies. Finally, we will provide an overview of the current body of knowledge on complex multiple-risk lifestyle interventions for reducing symptoms of depression. A meta-analysis will be performed and statistical integration of results will be used to compute common effect size and significance. The effect size, robustness and quality of evidence obtained in this meta-analysis will help to determine whether symptoms of depression can be reduced by complex multiple-risk lifestyle interventions based on the acquisition of healthy habits. The implication of the results obtained in this study is that complex multiple-risk lifestyle interventions could be routinely implemented in all patients at risk for depression in a variety of settings (ie, primary care centres, workplace, to name a few) and incorporated into treatment and prevention guidelines.

\section{Author affiliations}

${ }^{1}$ Department of Psychology, Universidad Loyola Andalucia, Seville, Spain

${ }^{2}$ Prevention and Health Promotion Research Network (redIAPP), ISCIII, Barcelona,

Spain

${ }^{3}$ Biomedical Research Institute of Malaga (IBIMA), Málaga, Spain

${ }^{4}$ Research Unit of the Health District of Primary Care Málaga-Guadalhorce, Málaga, Spain

${ }^{5}$ Centro de Investigación Biomédica en Red Fisiopatología de la Obesidad y Nutrición, Instituto de Salud Carlos III, Madrid, Spain

${ }^{6}$ Department of Family Medicine, Primary Care Division of Seville, Seville, Spain

${ }^{7}$ Institute of Biomedical Research of Salamanca (IBSAL), Primary Health Care Research Unit, La Alamedilla Health Center, Health Service of Castilla y León (SACyL), Salamanca, Spain

${ }^{8}$ Department of Public Health and Psychiatry, University of Málaga (UMA), Málaga, Spain

${ }^{9}$ El Palo Health Centre, Andalusian Health Service (SAS), Málaga, Spain

Contributors IGG is the guarantor. EM and JAB designed the study and the other authors (IGG, AR, PMP, SCC, MOC and JIR) collaborated in the design of the study. IGG prepared the initial protocol draft and EM and JAB revised the manuscript. All authors read, provided feedback, discussed and approved the final manuscript. All authors gave approval for the manuscript to be submitted.

Funding This study is supported by the Carlos III Health Institute, the Spanish Ministry of Economy and Competitiveness via a health research grant (PI15/01151; PI15/00762) through the Primary Care Prevention and Health Promotion Network (redlAPP, RD12/0005/0001; RD16/0007/0001), and by the EU ERDF funds (European Regional Development Fund).

Competing interests None declared.

Patient consent for publication Not required.

Provenance and peer review Not commissioned; externally peer reviewed.

Open access This is an open access article distributed in accordance with the Creative Commons Attribution Non Commercial (CC BY-NC 4.0) license, which permits others to distribute, remix, adapt, build upon this work non-commercially, and license their derivative works on different terms, provided the original work is properly cited, appropriate credit is given, any changes made indicated, and the use is non-commercial. See: http://creativecommons.org/licenses/by-nc/4.0/.

\section{REFERENCES}

1. World Health Organization (WHO). Depression. 2018 http://www.who. int/news-room/fact-sheets/detail/depression

2. Chisholm D, Sanderson K, Ayuso-Mateos JL, et al. Reducing the global burden of depression: population-level analysis of intervention cost-effectiveness in 14 world regions. Br J Psychiatry 2004;184:393-403.

3. Bellón JÁ, Moreno-Peral P, Motrico E, et al. Effectiveness of psychological and/or educational interventions to prevent the onset of episodes of depression: A systematic review of systematic reviews and meta-analyses. Prev Med 2015;76:S22-32.

4. Conejo-Cerón S, Moreno-Peral P, Rodríguez-Morejón A, et al. Effectiveness of psychological and educational interventions to prevent depression in primary care: a systematic review and metaanalysis. Ann Fam Med 2017;15:262-71.

5. van Zoonen K, Buntrock C, Ebert DD, et al. Preventing the onset of major depressive disorder: a meta-analytic review of psychological interventions. Int J Epidemiol 2014;43:318-29.

6. Deady M, Choi I, Calvo RA, et al. eHealth interventions for the prevention of depression and anxiety in the general population: a systematic review and meta-analysis. BMC Psychiatry 2017;17:1-14.

7. Tan L, Wang MJ, Modini M, et al. Preventing the development of depression at work: a systematic review and meta-analysis of universal interventions in the workplace. BMC Med 2014;12:74. 
8. Jacka FN, Berk M. Depression, diet and exercise. Med J Aust 2013;199:S21-3.

9. Sánchez-Villegas A, Delgado-Rodríguez M, Alonso A, et al. Association of the mediterranean dietary pattern with the incidence of depression: the seguimiento universidad de navarra/ University of Navarra follow-up (SUN) cohort. Arch Gen Psychiatry 2009;66:1090-8.

10. Almeida OP, Alfonso $\mathrm{H}$, Pirkis $\mathrm{J}$, et al. A practical approach to assess depression risk and to guide risk reduction strategies in later life. Int Psychogeriatr 2011;23:280-91.

11. Lopresti AL, Hood SD, Drummond PD. A review of lifestyle factors that contribute to important pathways associated with major depression: diet, sleep and exercise. J Affect Disord 2013;148:12-27.

12. Taylor G, McNeill A, Girling A, et al. Change in mental health after smoking cessation: systematic review and meta-analysis. BMJ 2014;348:g1151.

13. Mokdad AH, Marks JS, Stroup DF, et al. Actual causes of death in the United States, 2000. JAMA 2004;291:1238-45.

14. Galán I, Rodríguez-Artalejo F, Díez-Gañán L, et al. Clustering of behavioural risk factors and compliance with clinical preventive recommendations in Spain. Prev Med 2006;42:343-7.

15. Mozaffarian D, Afshin A, Benowitz NL, et al. Population approaches to improve diet, physical activity, and smoking habits: a scientific statement from the american heart association. Circulation 2012;126:1514-63.

16. Zhang QL, Zhao LG, Zhang W, et al. Combined impact of known lifestyle factors on total and cause-specific mortality among chinese men: a prospective cohort study. Sci Rep 2017;7:5293.

17. Sánchez-Villegas A, Martínez-González MA, Estruch R, et al. Mediterranean dietary pattern and depression: the PREDIMED randomized trial. BMC Med 2013;11:208

18. van der Meer RM, Willemsen MC, Smit F, et al. Smoking cessation interventions for smokers with current or past depression. Cochrane Database Syst Rev 2013;38:2-4.

19. Daley AJ, Foster L, Long G, et al. The effectiveness of exercise for the prevention and treatment of antenatal depression: systematic review with meta-analysis. BJOG: An International Journal of Obstetrics and Gynaecology 2015;122:57-62.

20. Dalgas $U$, Stenager $\mathrm{E}$, Sloth $\mathrm{M}$, et al. The effect of exercise on depressive symptoms in multiple sclerosis based on a meta-analysis and critical review of the literature. Eur J Neurol 2015;22:443-e34.

21. Herring MP. Effect of exercise training on depressive symptoms among patients with a chronic illness. Arch Intern Med 2012;172:101.

22. Brown JC, Huedo-Medina TB, Pescatello LS, et al. The efficacy of exercise in reducing depressive symptoms among cancer survivors: a meta-analysis. PLoS One 2012;7:e30955.

23. Mammen G, Faulkner G. Physical activity and the prevention of depression: a systematic review of prospective studies. Am J Prev Med 2013;45:649-57.

24. Psaltopoulou T, Sergentanis TN, Panagiotakos DB, et al Mediterranean diet, stroke, cognitive impairment, and depression: a meta-analysis. Ann Neurol 2013;74:580-91.

25. Molendijk M, Molero P, Ortuño Sánchez-Pedreño F, et al. Diet quality and depression risk: a systematic review and dose-response metaanalysis of prospective studies. J Affect Disord 2018;226:346-54

26. Zabaleta-Del-Olmo E, Pombo H, Pons-Vigués M, et al. Complex multiple risk intervention to promote healthy behaviours in people between 45 to 75 years attended in primary health care (EIRA study): study protocol for a hybrid trial. BMC Public Health 2018;18:874

27. Fine LJ, Philogene GS, Gramling R, et al. Prevalence of multiple chronic disease risk factors. 2001 National health interview survey. Am J Prev Med 2004;27:18-24.

28. Prochaska JJ, Spring B, Nigg CR. Multiple health behavior change research: an introduction and overview. Prev Med 2008;46:181-8.

29. Cezaretto A, Ferreira SR, Sharma S, et al. Impact of lifestyle interventions on depressive symptoms in individuals at-risk of, or with, type 2 diabetes mellitus: a systematic review and metaanalysis of randomized controlled trials. Nutr Metab Cardiovasc Dis 2016;26:649-62.

30. Coventry PA, Bower P, Keyworth C, et al. The effect of complex interventions on depression and anxiety in chronic obstructive pulmonary disease: systematic review and meta-analysis. PLoS One 2013;8:e60532.

31. Opie RS, O'Neil A, Itsiopoulos C, et al. The impact of whole-of-diet interventions on depression and anxiety: a systematic review of randomised controlled trials. Public Health Nutr 2015;18:2074-93.

32. Parekh S, Vandelanotte C, King D, et al. Improving diet, physical activity and other lifestyle behaviours using computer-tailored advice in general practice: a randomised controlled trial. Int $\mathrm{J}$ Behav Nutr Phys Act 2012;9:108-10.

33. Edington DW. Emerging research: a view from one research center. Am J Health Promot 2001;15:341-9.

34. King K, Meader N, Wright K, et al. Characteristics of interventions targeting multiple lifestyle risk behaviours in adult populations: a systematic scoping review. PLoS One 2015;10:1-13.

35. Tesler R, Harel-Fisch Y, Baron-Epel O. School health promotion policies and adolescent risk behaviour in Israel. $J$ Sch Health 2016;86:435-43.

36. Goetzel RZ, Ozminkowski RJ. The health and cost benefits of work site health-promotion programs. Annu Rev Public Health 2008;29:303-23.

37. Moher D, Shamseer L, Clarke M, et al. Preferred reporting items for systematic review and meta-analysis protocols (PRISMA-P) 2015 statement. Syst Rev 2015;4:1-9.

38. Higgins JPT GS. Cochrane handbook for systematic reviews of interventions. 5.1.0, 2011

39. Rychetnik L, Hawe P, Waters E, et al. A glossary for evidence based public health. J Epidemiol Community Health 2004;58:538-45.

40. Maderuelo-Fernandez JA, Recio-Rodríguez JI, Patino-Alonso MC, et al. Effectiveness of interventions applicable to primary health care settings to promote mediterranean diet or healthy eating adherence in adults: a systematic review. Prev Med 2015;76:S39-55.

41. Martínez-González MA, Salas-Salvadó J, Estruch R, et al. Benefits of the mediterranean diet: insights from the PREDIMED study. Prog Cardiovasc Dis 2015;58:50-60.

42. Khayyatzadeh SS, Mehramiz M, Mirmousavi SJ, et al. Adherence to a Dash-style diet in relation to depression and aggression in adolescent girls. Psychiatry Res 2018;259:104-9.

43. Sacks FM, Svrtkey LP, Vollmer WM, et al. Effects on blood pressure of reduced dietary sodium and the dietary approaches to stop hypertension (Dash) diet. N Engl J Med 2001;344:3-10.

44. Niu K, Momma H, Kobayashi $\mathrm{Y}$, et al. The traditional Japanese dietary pattern and longitudinal changes in cardiovascular disease risk factors in apparently healthy Japanese adults. Eur J Nutr 2016;55:267-79.

45. Suzuki T, Miyaki K, Tsutsumi A, et al. Japanese dietary pattern consistently relates to low depressive symptoms and it is modified by job strain and worksite supports. J Affect Disord 2013;150:490-8.

46. Food and Agriculture Organization and World Health Organization. Fruit and vegetables for health. 2004 http://www.who.int/ dietphysicalactivity/publications/fruit_vegetables_report.pdf.

47. World Health Organization (WHO). Diet, nutrition and the prevention of chronic diseases: World Health Organization technical report series, 2003.

48. World Health Organization. Fats and fatty acids in human nutrition. Report of an expert consultation: FAO food and nutrition paper, 2008.

49. World Health Organization (WHO). Global recommendations on physical activity for health. $2010 \mathrm{http}: / / \mathrm{www}$. who.int/ dietphysicalactivity/factsheet_recommendations/en/

50. Hughes JR, Keely JP, Niaura RS, et al. Measures of abstinence in clinical trials: issues and recommendations. Nicotine Tob Res 2003;5:13-26.

51. Fleiss JL, Levin B, Paik MC. Sons JW, Statistical methods for rates and proportions, 2013.

52. Higgins JPT. Assessing risk of bias in included studies. Higgins JPT GS, ed. Cochrane handbook for systematic reviews of interventions: Wiley, 2008:187-241.

53. Duval S, Tweedie R. Trim and fill: A simple funnel-plot-based method of testing and adjusting for publication bias in meta-analysis. Biometrics 2000;56:455-63.

54. Begg CB, Mazumdar M. Operating characteristics of a rank correlation test for publication bias. Biometrics 1994;50:1088-101.

55. Egger M, Davey Smith G, Schneider M, et al. Bias in meta-analysis detected by a simple, graphical test. BMJ 1997;315:629-34.

56. Cohen J, Hillsdale NJ. Statistical power analysis for the behavioral sciences. 2nd edn: Lawrence Earlbaum Associates, 1988:1-3.

57. D'agostino RB, Belanger A, D'Agostino Jr RB. A suggestion for using powerful and informative tests of normality Authors (s): Ralph B. D' Agostino, Albert Belanger, Ralph B. D' Agostino and Jr. Published by : Taylor \& Francis, Ltd. on behalf of the American Statistical Association Stable U. Am Stat 1990;44:316-21.

58. Knapp G, Hartung J. Improved tests for a random effects metaregression with a single covariate. Stat Med 2003;22:2693-710.

59. Higgins JP, Thompson SG. Controlling the risk of spurious findings from meta-regression. Stat Med 2004;23:1663-82.

60. Balshem $\mathrm{H}$, Helfand $\mathrm{M}$, Schünemann $\mathrm{HJ}$, et al. GRADE guidelines: 3. rating the quality of evidence. $J$ Clin Epidemiol 2011;64:401-6. 